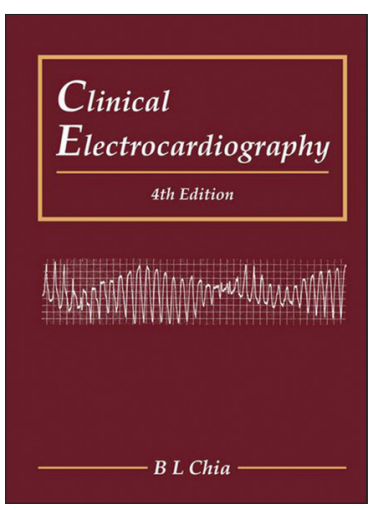

\title{
Clinical Electrocardiography 4th Edition
}

Author Chia Boon Lock

Publisher World Scientific Publishing Co Pte Ltd, Singapore

Published 2016

Pages 148, Paperback

ISBN 9789814723268

Retail price USD 29

Whether you are a medical student, house or medical officer, postgraduate resident, fellow in cardiology, cardiologist, coronary care unit/intensive care unit (CCU/ICU) nurse or a cardiac technician looking for a quick guide to understanding and interpreting electrocardiography (ECGs) in clinical practice, look no further. The $4^{\text {th }}$ Edition of Clinical Electrocardiography by Professor Chia Boon Lock, who is an Emeritus Professor at the National University of Singapore and an Emeritus Consultant at the National University Heart Centre Singapore, is a must-read that can turn a novice into a competent ECG interpreter.

The fourth edition has been updated to capture the latest and most up-to-date research on the interpretation of ECGs of various cardiac conditions and cardiac arrhythmias. Some ECG books are either too brief or so voluminous that the reader cannot follow or understand them. In less than 130 pages, Prof Chia uses his 45 years of clinical experience teaching clinical ECG to turn what has been deemed a difficult and complex subject into something very easy to understand. Each chapter is cleverly crafted, with ECG illustrations that have been meticulously scrutinised and selected from Prof Chia's lifelong personal collection to describe clues and diagnostic features of cardiac pathology, conditions and cardiac arrhythmias. The use of arrows and arrowheads to illustrate and highlight ECG abnormalities throughout the book makes identification and learning simple - the reader is immediately drawn to the relevant part of the ECG. In each figure, there are clear descriptions of the significant ECG characteristics of various conditions.

Chapter 1, on the normal ECG, is a must for every beginner who wants to learn how to record and read ECGs. It provides the fundamental principles and requirements, and can be extremely useful for medical undergraduates, cardiac technicians and CCU/ICU nurses. This is done in just 13 pages, a rare feat. In an era of reperfusion strategies (i.e. thrombolysis, acute coronary intervention for acute myocardial infarction [MI]) the 12-lead ECG has a pivotal role in the early diagnosis of various acute coronary syndromes and unstable angina (ST-segment elevation $\mathrm{MI}$ and non-ST-segment elevation MI).

In Chapter 2, Prof Chia takes a systematic, logical and clinical approach to the interpretation of acute MI ECG changes. Using clear ECG illustrations, he painstakingly explains the early hyperacute, transition, evolved and chronic phases of MI. Localisation of infarct sites is clearly explained. You can even pick up special gems of information on the scrutinisation of $T$ waves and $U$ waves. Chapter 3 tackles an equally important topic, the ability to differentiate ischaemic from nonischaemic ST-T wave changes, which include normal variants, early repolarisation changes, pericardial disease, ventricular hypertrophy, nonischaemic cardiomyopathy, acute pulmonary embolism, electrolyte disorders, intracranial haemorrhage and athlete's heart.

The next few chapters (Chapters 4-6) are dedicated to the principles and practice of interpreting cardiac arrhythmias. Prof Chia spells out two important basic principles that are paramount in assessing cardiac arrhythmias, highlighting the requirements for an accurate diagnosis and assessing the clinical setting in which the cardiac arrhythmia arises. The steps to take when assessing cardiac rhythm are highlighted, which is especially useful for practitioners who are required to read cardiac rhythms on monitor screens (i.e. residents, CCU/ICU nurses and advanced cardiac life support providers). The final chapter (Chapter 7), on bundle branch block and atrioventricular conduction defects, is highly relevant and can help doctors assess when to refer their patients to cardiologists for further evaluation, including those who require pacemaker implantation. One can read this manual from cover to cover in a few hours and be empowered with the knowledge, skills and confidence to make accurate and relevant ECG interpretations.

On a personal note, as I read and reviewed this book, I was reminded of my days as a medical student, resident and fellow in cardiology, when I had the privilege of being taught by the author. With this book, everyone will have the opportunity to learn ECG from a renowned cardiologist and Professor. This book deserves the most superlative description. I only regret that it does not come in a digital format (i.e. as an enhanced electronic book) that is compatible with PC and Macintosh computers, or with tablets and other mobile handheld devices. That would allow doctors and nurses to quickly reference it in the clinical setting to help them make better ECG interpretations and diagnoses, exercise the proper response, and save lives. 